\title{
Instrumentos para la promoción de la participación de la mujer en la ciencia: los premios L'Oréal-UNESCO en Argentina
}

\author{
NERINA FERNANDA SARTHOU*
}

Artículo recibido: 15 de abril de 2018

Artículo aprobado: 16 de agosto de 2018

Doi: http://dx.doi.org/10.12804/revistas.urosario.edu.co/desafios/a.6722

Para citar este artículo: Sarthou, N. F. (2019). Instrumentos para la promoción de la participación de la mujer en la ciencia: los premios L’Oréal-UNESCO en Argentina. Desafíos, 31(1), 83-120. Doi: http://dx.doi.org/10.12804/revistas.urosario.edu.co/desafios/a.6722

\section{Resumen}

En las últimas dos décadas aumentaron, significativamente, la cantidady la diversidad de instrumentos de política implementados en el ámbito de la ciencia, la tecnología y la innovación tanto en América Latina, como en Argentina. En este contexto, aunque se han creado nuevas iniciativas para la promoción de la participación de las mujeres en dicho ámbito, estas han sido pobremente incorporadas a los inventarios y diagnósticos en el área. Este trabajo analiza un tipo de instrumento especifico: los premios destinados, exclusivamente, a la mujer en la ciencia, en este caso, el Premio L'Oréal-UNESCO en Argentina. El enfoque se centra en tres aspectos principales desde el punto de vista

* Licenciada en Relaciones Internacionales de la Universidad Nacional del Centro de la Provincia de Buenos Aires (UNICEN), magíster en Estudios Latinoamericanos y doctora en Ciencia Política de la Universidad Nacional de General San Martín (UNSAM). Investigadora asistente del Consejo de Investigaciones Científicas y Técnicas (CONICET) y del Centro de Estudios Interdisciplinarios en Problemáticas Internacionales y Locales (CEIPIL/UNICEN), Centro Asociado a la Comisión de Investigaciones Científicas de la Provincia de Buenos Aires (СICPBA), Tandil, Argentina. Correo electrónico: nsarthou@ffch.unicen.edu.ar. OrCID: https://orcid.org/0000-0003-2671-4725 


\title{
84 / Nerina Fernanda Sarthou
}

tanto de la investigación, como de la formulación de politicas: las motivaciones de las premiadas para participar, los efectos que estas identifican, luego de ser distinguidas, y su opinión sobre la participación de la mujer en la ciencia en relación con los objetivos del premio. El estudio se sustenta en evidencia cualitativa mediante la combinación del análisis de documentos oficiales de los organismos patrocinadores y de la realización de entrevistas en profundidad a las ganadoras de los premios y distinciones.

Palabras clave: instrumentos de política pública, premio, género, ciencia, Argentina.

\section{Policy Instruments to Promote Women Participation in Science: The L'Oréal-UNESCO Prizes in Argentina}

\begin{abstract}
In the last two decades, the number and variety of policy instruments implemented in the field of science, technology and innovation in Latin America and particularly in Argentina has increased significantly. In this context, although new initiatives to promote the participation of women in the area have been created, they have been poorly incorporated into inventories and diagnoses in the area. This paper analyzes a specific type of policy instrument: prizes for women in science, in this case the L'Oréal-UNESCO prize in Argentina. The approach focuses on three main aspects from the point of view of both the research and the formulation of policies: the motivations of the winners to participate, the effects they identify after being distinguished and their opinion about the participation of women in science and its relation to the objectives of the award. The study is based on qualitative evidence through the combination of the analysis of official documents of the sponsoring agencies and the conduction of in-depth interviews with the prize winners.
\end{abstract} Keywords: Public policy instruments, prize, gender, science, Argentina. 


\title{
Instrumentos para a participação da mulher na ciência: os prêmios L'Oréal-UNESCO na Argentina
}

\begin{abstract}
Resumo
Nas últimas duas décadas aumentou significativamente a quantidade e a diversidade de instrumentos de política implementados no âmbito da ciência, a tecnologia e a inovação tanto na América Latina, como na Argentina. Neste contexto, ainda que se têm criado novas iniciativas para a promoção da participação das mulheres em dito âmbito, estas têm sido provavelmente incorporadas aos inventários e diagnósticos na área. Este trabalho analisa um tipo de instrumento especifico: os prêmios destinados exclusivamente à mulher na ciência, neste caso o prêmio L'Oréal-UNESCO na Argentina. O enfoque centra-se em três aspectos principais desde o ponto de vista tanto da pesquisa como da formulação de políticas: as motivações das premiadas para participar, os efeitos que estas identificam depois de ser distinguidas e, sua opinião sobre a participação da mulher na ciência em relação com os objetivos do prêmio. $O$ estudo sustenta-se em evidência qualitativa mediante a combinação da análise de documentos oficiais dos organismos patrocinadores e da realização de entrevistas em profundidade às ganhadoras dos prêmios e distinções.
\end{abstract}

Palavras-chave: instrumentos de política pública, prêmio, gênero, ciência, Argentina.

\section{Introducción}

En las últimas dos décadas, aumentaron, significativamente, la cantidad y la diversidad de instrumentos de política implementados en el ámbito de la ciencia, la tecnología y la innovación (СТI) en América Latina (Baptista, 2017) y en Argentina (Sarthou, 2018). En este contexto, han surgido distintas bases de datos que tienen como propósito reunir en un mismo sistema, de forma normalizada, la información acerca de las políticas y los instrumentos en CTI en América Latina.

$\mathrm{Al}$ explorar estas bases, considerando la perspectiva de género en las políticas CTI, se pudo constatar dos cuestiones: por un lado, que los instrumentos destinados a promover la participación en la CTI de mujeres y niñas se hallan escasamente identificados; por otro, 
que los premios, como tipo de instrumento, se encuentran subrepresentados en los inventarios. Tan solo la base de datos SPIN de la UNESCO contempla, dentro de los distintos tipos de beneficiarios, aquellos instrumentos destinados exclusivamente a mujeres. Allí es donde adquieren relevancia los premios L'Oréal-UNESCO "La Mujer y la Ciencia”, por ser un instrumento implementado en ocho países de la región y, en algunos casos, el único relevado a nivel nacional.

Además de estos indicios sobre una recopilación incompleta de las políticas que buscan fomentar la incorporación de las mujeres y de las niñas en las actividades de CTI, desde la noción de instrumentos de política, en la región se advierte que, a pesar de una mejora significativa, en especial en el número de mujeres que ingresan a carreras científicas (Franchi et al., 2008, Estébanez, 2007, 2012), varias de las estructuras organizativas de la ciencia y algunos científicos todavía se resisten a la plena participación de las mujeres (UNESCO, 2015).

Un vistazo panorámico de la situación de las mujeres en el principal organismo de gestión pública, en Argentina, para la formación y el desarrollo de la carrera científica, el Consejo Nacional de Investigaciones Científicas y Técnicas (CONICET) ${ }^{1}$ muestra que hubo un aumento contundente de la participación de la mujer entre 2007 y 2016: el número de investigadoras pasó de 2400 a 5338, lo que representa el $53 \%$ del total de los investigadores al final del periodo. A su vez, la cantidad de becarias (doctorales y posdoctorales) aumentó de 3363 a 6656 durante el mismo período, constituyendo el $60 \%$ del total de becarios del organismo. Este crecimiento en la proporción de mujeres que participan en CONICET se observa también en las categorías de la carrera del investigador del organismo aunque, como porcentaje del total de investigadores, las mujeres son minoría en la categoría más alta. Mientras en el año $2003^{2}$ las mujeres constituían el $40 \%$ de

\footnotetext{
1 Datos sección web "CONICET en CIFRAS". Recuperado de http://www.conicet.gov.ar/ acerca-de-conicet-en-cifras/

2 Los datos de 2003 fueron extraídos de la sección "Comunicación" del sitio del CONICET el 8 de marzo de 2015, Día Internacional de la Mujer. Recuperado de http:/ / www.conicet. gov.ar/mujeres-en-el-conicet-una-tendencia-creciente/
} 
los investigadores independientes, el $27 \%$ de los principales y solo el $18 \%$ de los superiores, en 2016 ya representaban el $49 \%$ de los investigadores independientes, el $39 \%$ de los principales y el $26 \%$ de los superiores. Como puede observarse, la presencia de mujeres en la categoría superior es significativamente dispar, a pesar de que las mujeres constituyen el $53 \%$ del total de investigadores del organismo.

Con base en lo señalado, este trabajo se propone explorar un instrumento de la política en CTI con rasgos particulares como son los premios y, a su vez, un premio exclusivamente destinado a las mujeres en ciencia. En particular, se abordan tres preguntas que son relevantes desde el punto de vista tanto de la investigación científica, como de la formulación de políticas: i) ¿Cuáles fueron las motivaciones de las premiadas para participar? ii) ¿Cuáles fueron los efectos que las entrevistadas identifican luego de ser distinguidas? iii) ¿Qué opinión tienen sobre la participación de la mujer en la ciencia quienes fueron premiadas y cómo su visión se relaciona con los objetivos del premio? Esta investigación utiliza evidencia cualitativa mediante la combinación del análisis de documentos oficiales de los organismos que otorgan el premio y de la realización de entrevistas en profundidad. Se entrevistó a las ganadoras del Premio L'Oréal-UneSCO-CONICET "Por las Mujeres en la Ciencia", tanto a las que fueron distinguidas con el premio como a las que recibieron una mención o una beca, a lo largo de las once ediciones. Se logró acordar una entrevista con la mayoría de las ganadoras, de allí que se cuente con el testimonio de veintidós premiadas, de este modo se pudo obtener al menos dos testimonios por cada una de las once ediciones.

La investigación busca revelar la dinámica de los premios y contribuir a una mejor comprensión de su potencial y de sus desventajas en un contexto nacional en el que se utilizan mecanismos más tradicionales para promover la participación de la mujer en la ciencia como lo son la licencia por maternidad, la prórroga para presentación de informes, la extensión de edad máxima según número de hijos, la disponibilidad de infraestructura (guarderías, jardines pertenecientes a universidades u otros organismos), entre otros. 
El artículo se estructura en tres secciones. En una primera, se presenta el estado del conocimiento sobre el tema. Primero, se realiza una presentación y caracterización de los premios en tanto instrumentos de política en CTI; luego, se da cuenta de las particularidades que presenta la problemática en género en materia de CTI y en relación con los premios. En una segunda sección, se realiza un recorrido por la introducción de la perspectiva de género en la UNESCO, para luego comprender el surgimiento de las versiones "nacionales" de los premios y del premio en Argentina. En una tercera parte se presentan los resultados y la discusión en torno a las entrevistas realizadas. Finalmente, se concluye con reflexiones conducentes a contribuir en términos académicos y de políticas públicas.

\section{Los premios como instrumentos de política en CTI}

En trabajos previos se ha realizado un recorrido por la literatura referida a la noción de instrumentos de política (Sarthou, 2015) y, más específicamente, se ha explorado el empleo de dicha noción en el marco del área de política en CTI en América Latina y en Argentina (Sarthou, 2018). Los instrumentos de política se han definido como mecanismos y técnicas para implementar o dar efecto a las políticas públicas (Salamon, 2002). Una clasificación tradicional es retomada por Vedung (2011) y consiste en identificar las vías a través de las cuales el gobierno busca influir en el comportamiento del sujeto y, en el grado de "obligación" de cada una, distingue así: la regulación, los medios económicos y la información, clasificación popularmente conocida con la expresión "garrotes, zanahorias y sermones". Los medios económicos, también conocidos como incentivos, varían si son formulados de forma positiva o negativa, es decir, si constituyen una entrega o una privación, y si son en efectivo o en especie. Los premios son un tipo de instrumento de incentivo (Vedung, 2011) mediante el cual se busca otorgar recompensas en efectivo o en especie para motivar a personas, grupos y comunidades a lograr diversos tipos de objetivos. Últimamente, los premios han atraído cada vez más la atención de legisladores, gerentes, filántropos y medios de comunicación debido a su potencial para inducir el desarrollo de la CTI y lograr objetivos relacionados con la recuperación económica o 
la participación de determinados grupos sociales en el desarrollo científico y tecnológico (Bays et al., 2009). Desde los estudios de la CTI, sin embargo, los premios han sido escasamente explorados a pesar de su larga historia y popularidad reciente.

Estos son uno de los tipos más antiguos de instrumentos de política en materia de CTI y no han sido empleados únicamente por los gobiernos sino que, con frecuencia, han sido una iniciativa de asociaciones científicas y empresas. Uno de los premios más conocidos en ciencia es el Premio Nobel creado, en 1895, y que se otorga anualmente para reconocer a personas o instituciones que hayan llevado a cabo investigaciones o contribuciones notables a la humanidad. Otros ejemplos históricos de premios para la innovación son: el Premio Longitud, creado por el Parlamento británico, en 1714, que condujo a importantes avances en materia naval; el premio francés para la preservación de alimentos durante las guerras napoleónicas; el Premio Orteig para un vuelo sin escalas entre Londres y Nueva York, y una serie de premios del Fondo Montyon (Gök, 2013). A pesar de la disminución de su uso durante el siglo Xx, al ser remplazados por becas o por el sistema de patentes, volvieron a ganar popularidad después de la década de los noventa mediante una serie de premios ofrecidos por diversas asociaciones y por los propios organismos gubernamentales.

Los premios están diseñados para reconocer ciertos logros definidos por quienes otorgan el premio como sobresalientes $y$, al mismo tiempo, para motivar a los destinatarios a seguir haciendo un trabajo excelente o, quizá, para desempeñarse aún mejor. Esta motivación puede extenderse a otras actividades y a otros individuos que aspiran a recibir el premio en el futuro (Frey \& Gallus, 2017). A pesar de que, en su mayoría, son otorgados por organizaciones benéficas o empresas y, en menor medida, por los gobiernos, se ha demostrado que las diferencias en términos de administración y diseño en los premios otorgados por estos diferentes patrocinadores son mínimas (Bays et al., 2009). Esta investigación busca explorar un tipo específico de premio, uno destinado exclusivamente a las mujeres y que se origina en una iniciativa conjunta entre una empresa multinacional, un organismo internacional y un organismo de gestión pública a nivel nacional. 
Los premios son solo uno de los enfoques utilizados para estimular la participación de la mujer en la ciencia. Otros instrumentos, mucho más empleados, incluyen la sanción de normativa, como es el caso de las licencias por maternidad o paternidad (García \& Morán, 2008); la infraestructura, como las guarderías o jardines que poseen algunas instituciones científicas o las propias universidades, e instrumentos más tradicionales, como subsidios para investigación, para asistencia a viajes y becas de formación exclusivas para mujeres (Comisión Europea, 2009). La mayor parte de los estudios sobre los premios se basa en comparaciones intuitivas entre los premios y los instrumentos más tradicionales para encontrar cuáles son los incentivos óptimos en determinadas circunstancias.

\section{La cuestión de género y los premios en el ámbito de la CTI}

Los estudios sobre las disparidades entre hombres y mujeres, respecto a la participación en la profesión científica, han sufrido transformaciones de acuerdo con los resultados que arrojan las investigaciones académicas, de un lado, y a la implementación de políticas públicas con perspectiva de género, del otro. En un trabajo reciente, Yáñez (2016) identifica tres enfoques políticos y de investigación que se desarrollaron de manera secuencial durante las décadas de los ochenta, los noventa y los dos mil y que aún permanecen vigentes y están estrechamente relacionados entre sí. Si acotamos la literatura al problema del desequilibro de la participación de la mujer frente a los hombres en la carrera científica, encontramos que su abordaje es relativamente reciente — años dos mil— y que pueden identificarse algunos interrogantes y temas clave para organizar el debate.

Una pregunta que se ha intentado explorar desde la literatura es: ¿por qué hay menos mujeres que hombres en la carrera científica ${ }^{3}$ ?

\footnotetext{
3 En la literatura sobre los factores que promueven $\mathrm{y} / \mathrm{u}$ obstaculizan las carreras profesionales de mujeres como investigadoras se hace referencia, especialmente, a los campos científicos dominados por hombres como son la ciencia, la tecnología, la ingeniería y las matemáticas (STEM).
} 
En muchos países se observa una paridad de género en la etapa de formación universitaria que se invierte al ingresar en los niveles iniciales de la profesión (doctorado) y se distancia a la par del ascenso de nivel. Este fenómeno se ha descrito en la literatura como el de "leaky pipeline" o "tubería con pérdidas o fugas", si se considera que el proceso de convertirse en investigador/a, en una sucesión de varias etapas educativas y laborales, es un conducto que tiene fugas: en cada momento de transición de un nivel educacional o profesional a otro se pierden más mujeres que hombres (Yáñez, 2016).

Un conjunto de trabajos afirma que el periodo de transición desde el posdoctorado hacia posiciones permanentes es una de las etapas de la carrera científica en que la deserción de las mujeres es particularmente elevada (Blickenstaff, 2005; Baader et al., 2017). Una de las causas encontradas se relaciona con la etapa de la "hora punta" (rush hour), momento en que las presiones laborales fuertes coinciden con los años fértiles de las mujeres y con las expectativas sociales sobre el momento adecuado para la maternidad, lo que afecta el desarrollo de la carrera científica (Mason et al., 2013). Esta situación se agrava en un contexto en el que las mujeres todavía son las principales responsables de los cuidados familiares y de las tareas domésticas (Misra et al., 2012), aun en el caso de parejas en las que ambos se dedican a la ciencia (Vohlídalová, 2017).

En el caso de Argentina, se realizó un estudio (Franchi et al., 2008) en el que se encontró que las mujeres que alcanzaron las categorías más altas en el sistema científico y tecnológico nacional, entre 1994 y 2006, en su mayoría, no formaron una familia. Si bien los autores reconocen no tener elementos para discernir si dicha situación fue una opción real o una imposición para el éxito en sus carreras, integran testimonios de mujeres prominentes del sector con edades entre los sesenta y setenta años que dijeron haber "decidido" no establecer una familia (casarse y tener hijos) por las presiones laborales.

Otras de las preguntas referidas a la participación de la mujer en la carrera científica es, ¿por qué hay menos mujeres en los estratos superiores o en cargos decisorios? Desde la literatura se relaciona 
con el fenómeno del techo de cristal o glass ceiling barriers (Bukstein \& Gandelman, 2017). Se refiere a la incapacidad que sufre la mujer para alcanzar puestos gerenciales, independientemente de su formación. Se denominó así ya que no hay ninguna ley que impida a la mujer avanzar en su trayectoria profesional, de allí que se trate de una barrera invisible difícil de traspasar. El techo de cristal aparece en la parte superior de la estructura jerárquica de la carrera científica limitando a las mujeres a alcanzar puestos con gran responsabilidad.

Entre las razones estudiadas, se ha llevado la atención al desempeño dispar entre hombres y mujeres en la carrera científica, es decir, se ha demostrado que la productividad (medida a través de la cantidad de publicaciones y citaciones) de las científicas es menor que la de los científicos (Nielsen, 2016). No obstante, se ha corroborado que las mujeres son tan productivas como sus colegas masculinos y aún más en algunos casos, cuando se toman en cuenta otros factores como las desiguales oportunidades de promoción y las actividades que no se publican (Mairesse \& Pezzoni, 2015) o las diferentes etapas de la carrera científica (van Arensbergen et al., 2012).

Otra de las razones abordadas han sido los sesgos de género en los criterios de evaluación y promoción en el ámbito científico, algunos culturales y otros institucionales. Estas forma de discriminación de género, por lo general, permanecen ocultas bajo el velo del ethos meritocrático que caracteriza a las instituciones académicas y que se relaciona con la estructura de poder de la ciencia (Yáñez, 2016). Los estudios realizados muestran, sin embargo, que la excelencia científica, vale decir, la definición y valoración de los méritos académicos como procesos en los cuales se sustenta el avance de una carrera, no es un "hecho universal", sino más bien una construcción social y, como tal, se abre a muchos tipos de sesgos, incluidos los sesgos de género. Estos repercuten en la evaluación de papers, de pedidos de subsidios y de promociones de categoría, afectando la productividad, la financiación y el ascenso a puestos jerárquicos de las mujeres en la ciencia.

Este tipo de sesgos ha conducido a la implementación de iniciativas que se denominan programas de acción afirmativa o de discriminación 
positiva. Estas intentan promover una representación equitativa de las mujeres en cargos de nivel superior, así como entre los miembros de la comunidad académica y científica. Entre ellas se encuentran las cuotas o cupos femeninos que garantizan una cierta fracción mínima de ganadoras y la norma de la repetición de la competencia a menos que se alcance un número crítico de ganadores femeninos en el primer intento (Balafoutas \& Sutter, 2012).

El sesgo de género en la financiación de la investigación ha sido demostrado por un Informe de la Comisión Europea (2009), aunque con una intensidad variable según país, disciplina y nivel organizativo. Por ejemplo, en los estados miembro de la Unión Europea y países asociados, los efectos de género en la financiación de la investigación se han identificado entre los solicitantes, receptores y guardianes (gatekeepers) del financiamiento de la investigación; en los procesos, instrumentos y criterios de financiación, y en el papel de las organizaciones clave para la igualdad en la investigación (Comisión Europea, 2009).

Vinculado al tema de la evaluación, emerge la cuestión de los premios y del género. Estos, al igual que las publicaciones, constituyen un antecedente importante en la trayectoria de un/a científico/a y pueden ser decisivos para la carrera — incluidas las decisiones de promoción y permanencia_-, a la vez que son otra medida para otorgar valor al trabajo científico y configurar las disciplinas. Las explicaciones al menor número de mujeres en la entrega de premios se basan en el "efecto embudo", es decir, como hay pocas mujeres con una sólida trayectoria científica, es difícil encontrar mujeres formadas, seleccionables y premiables. No obstante, a pesar de que ha habido un aumento sustancial en el número de mujeres que reciben doctorados en ciencias y tecnología, y las mujeres son una parte cada vez más importante de la fuerza laboral científica y profesional, la literatura concluye que las mujeres están significativamente subrepresentadas como receptoras de premios en el ámbito de la ciencia (Giménez Elorriaga \& Monike, 2016). Una creciente literatura documenta una serie de factores que ponen en desventaja a las mujeres para recibir premios, comenzando con la convocatoria de nominaciones (Lincoln et al., 2012). La investigaciones cuantitativas han probado, por un 
lado, que las mujeres se autopromocionan menos que los hombres y que no tienden a buscar ser nominadas o postuladas por otros (Rudman, 1998) y, por otro lado, que muchos premios evocan imágenes asociadas, estereotípicamente, con los hombres al describir los candidatos apropiados, a menudo utilizando un lenguaje como "líderes" o personas que "toman riesgos" (Carnes et al., 2005).

Después de que una nominación se envía a un comité de adjudicación, los procesos demográficos pueden influir en el proceso de evaluación. Por ejemplo, Lincoln y colaboradores (2012) encontraron que, en una sociedad de física, la presencia de cada mujer en un comité de premios duplicaba las posibilidades de que una mujer ganara un premio y que los comités presididos por mujeres tenían tres veces más probabilidades que los presididos por hombres de nombrar a una mujer como ganador.

La disparidad de las mujeres como ganadoras de premios es una muestra evidente del efecto Matilda en la ciencia. Fue el sociólogo Robert Merton quien denominó y estudió el "efecto Mateo" en la ciencia a colación de una parábola del evangelio según San Mateo que dice: "a todo el que tiene se le dará y le sobrará, pero al que no tiene, aun lo que tiene se le quitará”. Merton estudió la manera en la que esta parábola funcionaba en la ciencia, encontrando que los investigadores que tenían más recursos, mejores cargos, más proyectos, más citas, más premios eran los que más de estos recursos recibían, dificultando que los investigadores que empezaban su carrera accedieran de manera equilibrada al sistema de recompensas. La historiadora de la ciencia Margaret Rossiter (1993) dio una vuelta a este teorema al estudiar el efecto Matilda según el cual, en ese escenario descrito por Merton, las mujeres son aún más vulnerables al efecto Mateo, es decir, las mujeres y sus contribuciones científicas se acreditan a los hombres —lo que tienen se les quitará— o se pasan por alto por completo ${ }^{4}$.

4 Lo llamó así en honor a Matilda Gage, sufragista neoyorkina de finales del siglo XIX que identificó y denunció la invisibilización de las mujeres y de sus méritos en otros contextos. 
A pesar de esta disparidad referida a los premios en la ciencia y la cuestión de género, es relativamente reciente la literatura al respecto, más aún de los premios destinados solo a mujeres. En general, los trabajos abordan los premios en una disciplina en particular, por ejemplo, Silver y colaboradores (2017) concluyen que, desde 1948 en adelante, las médicas han estado subrepresentadas entre los 264 premios que entrega periódicamente la Academia Estadounidense de Medicina Física y Rehabilitación. Otras obras son las de Giménez Elorriaga y Monike (2016) sobre el Premio Ada Byron, de la Universidad de Deusto, en el País Vasco, destinado a mujeres tecnólogas, y el de Weir y colaboradores (2014) sobre la dinámica de la Beca Internacional L’Oréal, destinada a científicas jóvenes.

En el caso de Argentina, un estudio sobre el periodo 2003 a 2007 acerca de la distribución por género de cuatro premios destacados en el país encontró que todos ellos se encontraban masculinizados y algunos, como es el caso del de la Academia Nacional de Derecho y Ciencias Sociales, estaban absolutamente masculinizados (Baringoltz \& Posadas, 2007). Los autores afirmaron, además, que no existe ninguna academia, premio o reconocimiento en que participen en igualdad de oportunidad varones y mujeres que se encuentre feminizado.

\section{La perspectiva de género como prioridad global de la UNESCO}

La UNESCO ${ }^{5}$ se creó en 1945 en el seno de la Organización de las Naciones Unidas con el propósito de contribuir a la paz y a la seguridad estrechando, mediante la educación, la ciencia y la cultura, la colaboración entre las naciones. Lejos estuvo en su momento la incorporación de la cuestión de género entre sus acciones; no obstante, la propia evolución del sistema internacional y de la agenda mundial convirtió

\footnotetext{
5 Actualmente posee 195 Estados miembro y se estructura en torno a una Conferencia General, un Consejo Ejecutivo (58 miembros) y una Secretaría. Su máxima autoridad es el director general que, en 2009, resultó ser por primera vez una mujer: Irina Bokova. En 2017 fue elegida nuevamente una mujer, Audrey Azoulay.
} 
al organismo en un actor protagonista, a escala global, respecto a la situación de mujeres y niñas.

La labor de la UNESCO se organiza a través de sus cinco programas mayores: i) Educación; ii) Ciencias naturales y exactas; iii) Ciencias sociales y humanas; iv) Cultura y Comunicación; y v) Información, y el enfoque de sus acciones se define mediante un documento denominado "Estrategia a plazo medio". Fue en la "Estrategia 2008-2013" donde se establecieron, por primera vez, de manera explícita, las dos prioridades globales de la UNESCO que se mantienen hoy en día y son transversales a los distintos ejes temáticos que aborda la organización: África y la igualdad de género. Dichas prioridades globales fueron recomendadas por el documento final de la Cumbre Mundial de 2005, donde también tuvo origen un proceso de reforma orientado a todo el sistema de Naciones Unidas a nivel mundial, regional y nacional (UNESCO, 2007). Actualmente se encuentra en vigencia la "Estrategia a plazo medio 2014-2021" que retoma las dos prioridades globales ya establecidas —África y la igualdad de género-y distingue dos grandes objetivos generales: la cultura de paz y el desarrollo sostenible. A su vez, establece nueve objetivos estratégicos que no se vinculan de manera exclusiva y unidimensional a ningún programa mayor en particular, sino que exigen la intervención y colaboración de varios programas en forma interdisciplinaria (UNESCO, 2014a).

La "Estrategia a plazo medio 2014-2021" fue acompañada del "Plan de acción prioritario de igualdad de género de la UNESCO 2014-2021”. Dicho plan explica qué significa la igualdad de género para la UNESCO y proporciona orientación sobre cómo la organización garantizará que la perspectiva de género se refleje en todas sus políticas, programas y procesos para que la igualdad de género avance tanto dentro de los procesos institucionales de la Secretaría, como en su trabajo con Estados miembro. De este modo, para la UNESCO, la noción de igualdad de género significa igualdad de derechos, responsabilidades y oportunidades tanto para mujeres y hombres como para niñas y niños. Supone que se tengan en cuenta los intereses, necesidades y prioridades de mujeres y hombres, reconociendo la diversidad de los distintos grupos de personas. La igualdad 
de género es un principio relativo a los derechos humanos, un prerrequisito para un desarrollo sostenible centrado en las personas y un objetivo en sí misma (UNESCO, 2014b). Con el fin de lograr resultados concretos y sostenibles para la promoción de la igualdad de género, la UNESCO aplica un doble enfoque: (i) incorporación de la perspectiva de género en todos los programas y actividades; y (ii) programas específicos para abordar la cuestión de género.

Los programas dirigidos a cuestiones de género tienen como objetivo reducir las desigualdades concretas que enfrentan mujeres u hombres, niñas o niños en una situación particular. El objetivo de este enfoque es abordar casos específicos y significativos de discriminación y reducir las desigualdades mediante el apoyo a un grupo en particular. Esto incluye, por ejemplo, actividades dirigidas al empoderamiento de la mujer en situaciones en que sufren formas particulares de desigualdad y discriminación. Entre los principales programas actuales de género que posee la UNESCO se encuentra el programa L'OréalUNESCO "Mujeres en la Ciencia".

Dicho programa fue una iniciativa de la empresa francesa de cosméticos L'Oréal ${ }^{6}$ y de UNESCO una década antes de que la igualdad de género fuera incorporada a documentos oficiales como prioridad global del organismo. Desde 1998, se otorgan los premios L'OréalUNESCO, en el marco del mencionado programa, consistentes en la premiación anual de cinco mujeres que hayan realizado contribuciones importantes al desarrollo de la ciencia. Los premios se conceden alternando dos años en Ciencias de la Vida y uno en Ciencias de la Materia y se convocan cinco premios de acuerdo a las regiones geográficas: África y Oriente Medio, Asia-Pacífico, Latinoamérica ${ }^{7}$ y el Caribe, y Norteamérica.

\footnotetext{
6 El grupo L'Oréal, con la sede de su casa matriz en Clichy, Francia, es la compañía de cosméticos más grande del mundo. Desde su fundación, en 1909, por el químico y empresario Eugène Schueller, con uno de los primeros tintes para cabellos, la empresa y sus innovaciones han tenido un éxito indiscutido.

7 Entre las argentinas distinguidas se encuentran Mariana Weissmann (2003), Cecilia Bouzat (2013) y Amy Austin (2018).
} 
Además de los cinco premios, en el año 2000 se puso en marcha el programa de becas internacionales UNESCO-L'Oréal ${ }^{8}$ que presta apoyo a trabajos de jóvenes científicas prometedoras que cursan estudios de doctorado o posdoctorado en todo el mundo. El programa otorga una beca, de dos años de duración, de 40000 dólares para ayudar en la financiación de sus trabajos de investigación en curso, en Ciencias de la Vida o Ciencias de la Materia, según la edición, y ofrece la oportunidad de continuar los estudios en una institución científica de un país extranjero. Para garantizar la diversidad del mismo, las becas se atribuyen cada año a tres mujeres de las cinco regiones.

Desde 2015, está en marcha el programa UNESCO-L'Oréal Talentos Emergentes ${ }^{9}$ que premia a quince jóvenes de carrera prometedora, tres por cada región, seleccionadas entre las becadas en los programas nacionales. El programa tiene un doble objetivo: por un lado, reconocer y dar visibilidad a la carrera de investigación de mujeres científicas excepcionales cuyo trabajo haya contribuido al avance del conocimiento científico en beneficio de la sociedad; por el otro, fomentar la vocación científica entre las jóvenes.

Hacia 2006, UNESCO y L’Oréal, en colaboración con organismos científicos gubernamentales, comenzaron a promover premios y becas nacionales en distintos países de América Latina. En Brasil, desde 2006, se entrega el Premio L'Oréal Brasil, en conjunto entre UnESCO Brasil y la Academia Brasileña de Ciencias. En 2007, además de Argentina, fueron México, Chile y Uruguay los países con iniciativas similares. En México, desde 2007, se abren las convocatorias "Becas para las Mujeres en la Ciencia" a partir de la colaboración entre L'Oréal México, la Comisión Mexicana de Cooperación con la UnesCo, la Oficina de la UnESCO en México, el Consejo Nacional de Ciencia y Tecnología y la Academia Mexicana de Ciencias. En Chile, también desde 2007, se entrega el Premio L'Oréal Chile-UnESCO, con

\footnotetext{
8 Entre las argentinas ganadoras se encuentran María Gabriela Palomo (2003), Carolina Trochine (2008) y Paula Villar (2009).

9 En 2017, entre las quince premiadas, se distinguió a la argentina Julia Etulain, ganadora de la beca nacional en 2015 .
} 
el patrocinio de la Comisión Nacional de Investigación Científica y Tecnológica. En Uruguay, L’Oréal y UNESCO, junto con el Ministerio de Educación y Cultura y la Agencia Nacional de Investigación e Innovación, entregan el Premio L'Oréal-Unesco Uruguay. En 2008, se lanzó en Colombia el programa nacional de becas L'OréalUNESCO que tiene por objetivo premiar el mérito científico y aumentar el número de mujeres colombianas en el ámbito investigativo. Ese mismo año, en Perú, también comenzó a entregarse el premio a partir de convocatorias surgidas desde L'Oréal Perú, la Oficina de UNESCO en Lima y el Consejo Nacional de Ciencia, Tecnología e Innovación Tecnológica.

A diferencia de otros premios patrocinados por academias de ciencias, asociaciones científicas disciplinares u organismos científicos nacionales, los premios mencionados son entregados por una empresa de cosméticos debido a su reconocimiento por un alto nivel de inversión en investigación y desarrollo $(\mathrm{I}+\mathrm{D})$. De allí que estos premios no sean una iniciativa aislada sino que L'Oréal ha ido convocando a diferentes instituciones dedicadas a la investigación a ser socias de sus diversos programas ${ }^{10}$. No obstante, a pesar de la legitimidad ${ }^{11} \mathrm{y}$ aceptación entre la comunidad científica que han adquirido los distintos premios L'Oréal, en el imaginario social el binomio cienciabelleza puede generar representaciones que afecten la validez de los premios en las sociedades locales, al no ser una institución asociada a la actividad científica, y ello puede repercutir sobre la imagen de mujer científica que se busca difundir y promover.

10 En el Informe anual 2015 de la fundación se encuentra un listado de socios, entre los que se mencionan universidades, laboratorios, institutos de hospitales, academias de ciencias y demás asociaciones de la sociedad civil vinculadas a la investigación. Recuperado de www.fondationloreal.com/documents/-90f17f6a-5802-4710-869f-7b0ca43066ad/ download?lang=en

11 La alta calidad exigida por los premios L'Oréal-UNESCO quedó demostrada a partir de que los trabajos de dos galardonadas por el programa, en 2008, fueron reconocidas con el Premio Nobel en 2009. Ada E. Yonath recibió el Premio Nobel de Química en 2009, compartido con Venkatraman Ramakishnan y Thomas A. Steitz. Por su parte, Elizabeth Blackburn recibió el Premio Nobel de Medicina 2009, junto a Carol W. Greider y Jack W. Szostak (López Sancho, 2016). 


\section{Los premios L'Oréal-UNESCO en Argentina}

En Argentina, existen diversos premios específicos, destinados a reconocer trayectorias científicas o promover proyectos de investigación, otorgados por academias o entes gubernamentales. Entre ellos pueden mencionarse los premios de la Academia Nacional de Ciencias Exactas, Físicas y Naturales, la Academia Nacional de Medicina, la Academia Nacional de Ciencias de la provincia de Córdoba y la Distinción Investigador/a de la Nación, con los premios Houssay, Houssay Trayectoria y Jorge Sábato. Asimismo, existe otro tipo de premios que son entregados por empresas en asociación con algún organismo gubernamental, como el Premio Arcor a la Innovación ${ }^{12}$ y el Premio Tenaris al desarrollo tecnológico argentino ${ }^{13}$, ambos entregados en conjunto con la Agencia Nacional de Promoción Científica y Tecnológica (ANPCYT). Si bien entre los ganadores de estos diversos premios pueden encontrarse algunas mujeres, ninguno de ellos está destinado exclusivamente a la participación de la mujer.

En este contexto nacional, en 2007, se crea el Premio L'Oréal-UNESCO "Por las Mujeres en la Ciencia", con la colaboración de CONICET. Como su nombre lo indica, es otorgado por un organismo científico gubernamental, como lo es CONICET, la sede de la empresa L'Oréal en Argentina y la Comisión Permanente de Unesco en el país $\left(\right.$ CONAPLU $\left.^{14}\right)$. En términos de gestión del premio, los primeros dos organismos son quienes poseen una presencia más activa: mientras L’Oréal aporta los recursos económicos, participa de la instancia de evaluación de las candidatas y — especialmente- difunde a las ganadoras y sus proyectos, CONICET se encarga de la difusión de la convocatoria, recepción de solicitudes y gestión del proceso de evaluación.

\footnotetext{
12 Para mayor información sobre este premio ver http://www.arcorinnovacion.com/

13 Para mayor información sobre este premio ver http://www.tenaris.com/premiotenaris/ default.asp

14 Creada en 1958, y es una comisión gubernamental cuya Secretaría Ejecutiva funciona en el Ministerio de Educación y Deportes de la Nación, en el marco de la Dirección Nacional de Cooperación Internacional, siendo el ministro de Educación quien la preside. La comisión es un órgano colegiado integrado por dependencias gubernamentales con influencia en los diferentes campos de UNESCO.
} 
Según los documentos de la última convocatoria, que tuvo lugar en $2017^{15}$, el objetivo general declarado " [...] se funda en el postulado de la UNESCO que sostiene que las personas son lo más importante y que la innovación y la excelencia, sobre todo cuando se da en los ambientes científicos, merecen ser fomentadas mediante esfuerzos concertados de toda la comunidad nacional. Este premio [...] pretende coadyuvar a la continuidad de proyectos ya existentes y en curso de ejecución, y colaborar con la formación de jóvenes de gran potencial. También pretende consolidar el papel de la mujer en la concepción de soluciones científicas a los problemas con que debe enfrentarse la sociedad en el nuevo milenio" (Bases Premio L'Oréal-UnesCo-Conicet, 2017).

El diseño del premio, al ser exclusivamente abierto a la participación de las mujeres, combate los problemas asociados al efecto Matilda (Rossiter, 1993) ya que únicamente tiene en cuenta las contribuciones científicas de las mujeres. El objetivo del premio es identificar y recompensar un proyecto de un grupo de investigación cuya directora sea una mujer, especializada en el área del conocimiento definido para cada año (Ciencias de la Vida o Ciencias de Materiales) que se destaque por su dedicación, compromiso, beneficios, aporte al desarrollo de la investigación e impacto en Argentina. Por su parte, la beca tiene por objetivo identificar y recompensar a una becaria posdoctoral o investigadora joven de alto potencial, con cierta edad y proveniente de determinada área del conocimiento definida cada año, que se destaque por su dedicación, compromiso, beneficios, aporte al desarrollo de la investigación e impacto en Argentina. De este modo, la presentación que cada postulante debe hacer consta de un proyecto de investigación y de su curriculum vitae.

Un rasgo relevante del premio es que implica recursos económicos para el desarrollo de un proyecto de investigación. Entre 2007 y 2013, el

\footnotetext{
15 Los requisitos para participar han sufrido pequeñas variaciones a lo largo de las once ediciones En la primera edición, año 2007, se entregó un único premio, pero ya en 2008, y hasta 2014, se entregaron un premio y dos menciones especiales. En las ediciones de 2015 y 2016 se entregó un premio y una beca, mientras que en 2017 se entregaron seis distinciones (un premio y dos menciones y una beca y dos menciones). Las menciones en la última edición no estuvieron acompañadas de recursos económicos.
} 
monto consistió en la suma de 20000 dólares estadounidense para las ganadoras del premio; luego esa cifra se tradujo en pesos argentinos, aumentando año tras año para intentar mantener su valor en relación con la divisa. Es de destacar que, en el contexto de los instrumentos de financiamiento de la investigación en Argentina, los recursos económicos asociados al premio y la beca de L'Oréal-UNESCO son de singular importancia ya que, en general, han superado el monto que representa un subsidio otorgado mediante un Proyecto de Investigación Científica y Tecnológica (PICT) ${ }^{16}$ adjudicado por la ANPCYT, principal instrumento de financiamiento de la investigación en el país, no solo por la magnitud de los montos, sino por su estabilidad en el tiempo (Suárez \& Fiorentin, 2018).

La selección del jurado es una instancia decisiva dentro de la implementación de este instrumento. Como se mencionó, es CONICET el que dispone de personal para que se dedique a organizar el proceso. El mismo consiste en dos etapas: primero se envían las postulaciones a pares evaluadores y se selecciona un total de diez proyectos que pasan a una segunda etapa en donde un tribunal de notables toma la decisión final. Dicho tribunal ha estado constituido, en general, por el presidente o un representante del directorio de CONICET, uno o dos científicos o científicas de alto reconocimiento, alguna ganadora del premio en ediciones anteriores, en ocasiones un representante de la CONAPLU y el director general de L'Oréal Argentina.

\footnotetext{
16 Por ejemplo, en 2016, el premio y la beca consistieron en 250000 y 150000 pesos argentinos (para desarrollar un proyecto durante un año), respectivamente. Por su parte, los PICT categoría (D) Equipo de Trabajo de Reciente Formación (pueden presentarlo investigadoras menores de 48 años, con una trayectoria que se asemeja al de las ganadoras del premio) consistió en 130000 pesos argentinos (primer desembolso anual para proyecto de tres años) y la categoría (B) PICT Joven (científicos/as menores de 38 años, perfil similar al de las ganadoras de las becas) consistió en 85000 pesos argentinos (primer desembolso anual para proyecto de dos años). En 2017, los montos de L'Oréal-UNESCO fueron de 300000 (premio) y 180000 (beca) pesos argentinos, mientras que los PICT otorgaron en categoría D 155000 y en categoría B 105000 pesos argentinos. Como puede advertirse, el monto del premio y la beca superan significativamente -al menos en 2016 y 2017 - el monto anual de un PICT D y B, respectivamente.
} 
A continuación se presenta, primero, una caracterización sociodemográfica de las premiadas entrevistadas (tabla 1) y, en segundo lugar, tres dimensiones analizadas a partir de las entrevistas realizadas.

En primer lugar, se puede decir que todas las premiadas pertenecen a una misma clase social debido a su condición de científicas. En relación con un tema discutido en la literatura como lo es la cuestión familiar (Misra et al., 2012) y la maternidad (Mason et al., 2013), entre las diecinueve entrevistadas ${ }^{17}$, dieciocho son madres, mientras que dieciocho se encuentran en pareja (en la mitad de los casos la pareja ejerce también la profesión de científico), es decir, todas las entrevistadas han constituido una familia a la vez que han logrado llevar adelante o están construyendo — en el caso de las más jóvenes — una carrera científica.

Con relación a la edad de las ganadoras, la iniciativa está diseñada para afectar dos grupos de científicas con problemas distintos al momento de continuar su carrera profesional: de un lado, las ganadoras del premio (o distinción asociada al premio) son científicas con un puesto permanente como investigadoras de CONICET que se encuentran en una fase de proceso de consolidación de su carrera y que tienen entre 42 y 49 años (Yáñez, 2016; Bukstein \& Gandelman, 2017). De otro lado, las ganadoras de las becas (o distinciones asociadas a la beca) son becarias posdoctorales que se encuentran en una situación incierta y precaria de su carrera científica y tienen entre 32 y 34 años (Blickenstaff, 2005; Baader, et al., 2017).

17 En 2017, se entregaron, además, dos menciones en el marco del premio a Valeria Levi, investigadora independiente del CONICET en el Instituto de Química Biológica de la Universidad de Buenos Aires y a Andrea Quiberoni, investigadora independiente del CONICET en el Instituto de Lactología Industrial de la Universidad Nacional del Litoral, y dos menciones en el marco de la beca a Natalia Servetto, becaria posdoctoral del CONICET en el Instituto de Diversidad y Ecología Animal de la Universidad Nacional de Córdoba y a Natalia Andersen, investigadora asistente del CONICET en el Instituto de Investigaciones Bioquímicas de la Universidad Nacional del Sur. Dichas menciones no estuvieron acompañadas de un subsidio económico, con lo cual se diferencian de todos los casos anteriores en un aspecto teórico y metodológico importante para el desarrollo de esta investigación; de allí que se tomara la decisión de no incorporar el testimonio de las ganadoras de las menciones en esta ocasión. 
Tabla 1. Listado de científicas argentinas entrevistadas ${ }^{18}$

\begin{tabular}{|c|c|c|c|c|}
\hline $\begin{array}{l}\text { Año de } \\
\text { entrega }\end{array}$ & Distinción & Entrevistadas & $\begin{array}{c}\text { Lugar de trabajo al momento } \\
\text { de recibir el premio }\end{array}$ & Disciplina \\
\hline 2007 & Premio & $\begin{array}{l}\text { Cecilia } \\
\text { Bouzat }\end{array}$ & $\begin{array}{l}\text { Instituto de Investigaciones } \\
\text { Bioquímicas/CONICET- } \\
\text { Universidad Nacional del Sur }\end{array}$ & Bioquímica \\
\hline \multirow[t]{2}{*}{2008} & \multirow[t]{2}{*}{ Mención } & $\begin{array}{l}\text { Karen } \\
\text { Hallberg }\end{array}$ & $\begin{array}{l}\text { Instituto de Nanotecnología } \\
\text { y Nanociencia/ Comisión } \\
\text { Nacional de Energía } \\
\text { Atómica }\end{array}$ & Física \\
\hline & & $\begin{array}{l}\text { Patricia } \\
\text { Tissera }\end{array}$ & $\begin{array}{l}\text { Instituto de Astronomía y } \\
\text { Física del Espacio/CONICET- } \\
\text { Universidad de Buenos Aires }\end{array}$ & Física \\
\hline \multirow[b]{2}{*}{2009} & \multirow[b]{2}{*}{ Mención } & $\begin{array}{l}\text { Jorgelina } \\
\text { Ottado }\end{array}$ & $\begin{array}{l}\text { Instituto de Biología Mole- } \\
\text { cular y Celular/CONICET } \\
\text { Universidad Nacional de } \\
\text { Rosario }\end{array}$ & Agronomía \\
\hline & & $\begin{array}{l}\text { Fernanda } \\
\text { Drincovich }\end{array}$ & $\begin{array}{l}\text { Centro de Estudios } \\
\text { Fotosintéticos y } \\
\text { Bioquímicos/CONICET } \\
\text { Universidad Nacional de } \\
\text { Rosario }\end{array}$ & Bioquímica \\
\hline \multirow{3}{*}{2010} & Premio & $\begin{array}{l}\text { Patricia } \\
\text { Tissera }\end{array}$ & $\begin{array}{l}\text { Instituto de Astronomía y } \\
\text { Física del Espacio/CONICET- } \\
\text { Universidad de Buenos Aires }\end{array}$ & Astronomía \\
\hline & \multirow{2}{*}{ Mención } & $\begin{array}{l}\text { Adriana } \\
\text { Serquis }\end{array}$ & $\begin{array}{l}\text { Departamento de } \\
\text { Caracterización de } \\
\text { Materiales/ Comisión } \\
\text { Nacional de Energía } \\
\text { Atómica }\end{array}$ & Física \\
\hline & & $\begin{array}{l}\text { Miriam } \\
\text { Castro }\end{array}$ & $\begin{array}{l}\text { Instituto de Investigaciones } \\
\text { en Ciencia y Tecnología } \\
\text { de Materiales/CONICET- } \\
\text { Universidad Nacional de Mar } \\
\text { del Plata }\end{array}$ & $\begin{array}{l}\text { Ingeniería } \\
\text { Química }\end{array}$ \\
\hline
\end{tabular}

18 En tres ocasiones, la ganadora del premio fue distinguida con una mención en una edición anterior del galardón, de allí que, mientras el número de entrevistadas asciende a diecinueve, la totalidad de instancias sobre las cuales se obtuvo información fueron veintidós (tabla 1). 


\begin{tabular}{|c|c|c|c|c|}
\hline $\begin{array}{l}\text { Año de } \\
\text { entrega }\end{array}$ & Distinción & Entrevistadas & $\begin{array}{l}\text { Lugar de trabajo al momento } \\
\text { de recibir el premio }\end{array}$ & Disciplina \\
\hline \multirow{2}{*}{2011} & Premio & $\begin{array}{l}\text { María } \\
\text { Fernanda } \\
\text { Ceriani }\end{array}$ & Fundación Instituto Leloir & Bioquímica \\
\hline & Mención & $\begin{array}{l}\text { María Euge- } \\
\text { nia Farias }\end{array}$ & $\begin{array}{l}\text { Planta Piloto de Procesos } \\
\text { Industriales Microbiológicos, } \\
\text { CONICET-Tucumán }\end{array}$ & Biología \\
\hline \multirow[t]{2}{*}{2012} & \multirow[t]{2}{*}{ Mención } & $\begin{array}{l}\text { Mónica } \\
\text { Quiroga }\end{array}$ & $\begin{array}{l}\text { Instituto de Investigaciones } \\
\text { en Catálisis y Petroquímica/ } \\
\text { CONICET-Universidad } \\
\text { Nacional del Litoral }\end{array}$ & Química \\
\hline & & $\begin{array}{l}\text { Silvia } \\
\text { Goyanes }\end{array}$ & $\begin{array}{l}\text { Instituto de Física/CONICET- } \\
\text { Universidad de Buenos Aires }\end{array}$ & Física \\
\hline \multirow{2}{*}{2013} & Premio & $\begin{array}{l}\text { Fernanda } \\
\text { Drincovich }\end{array}$ & $\begin{array}{l}\text { Centro de Estudios } \\
\text { Fotosintéticos y Bioquímicos/ } \\
\text { CONICET-Universidad } \\
\text { Nacional de Rosario }\end{array}$ & Bioquímica \\
\hline & Mención & $\begin{array}{l}\text { Mariana } \\
\text { Maccioni }\end{array}$ & $\begin{array}{l}\text { Centro de Investigaciones en } \\
\text { Bioquímica Clínica e Imuno- } \\
\text { logia/CONICET-Universidad } \\
\text { Nacional de Córdoba }\end{array}$ & Química \\
\hline \multirow{2}{*}{2014} & Premio & $\begin{array}{l}\text { Adriana } \\
\text { Serquis }\end{array}$ & $\begin{array}{l}\text { Departamento de } \\
\text { Caracterización de } \\
\text { Materiales/Comisión } \\
\text { Nacional de Energía } \\
\text { Atómica }\end{array}$ & Física \\
\hline & Mención & Silvia Ceré & $\begin{array}{l}\text { Instituto de Investigaciones } \\
\text { en Ciencia y Tecnología } \\
\text { de Materiales/CONICET- } \\
\text { Universidad Nacional de Mar } \\
\text { del Plata }\end{array}$ & Química \\
\hline \multirow[t]{2}{*}{2015} & Premio & Amy Austin & $\begin{array}{l}\text { Instituto de Investigaciones } \\
\text { Fisiológicas y Ecológicas } \\
\text { vinculadas a la Agricultura/ } \\
\text { Universidad de Buenos Aires }\end{array}$ & Agronomía \\
\hline & Beca & Julia Etulain & $\begin{array}{l}\text { Instituto de Biología y } \\
\text { Medicina experimental/ } \\
\text { CONICET }\end{array}$ & Bioquímica \\
\hline
\end{tabular}




\begin{tabular}{|c|l|l|l|l|}
\hline $\begin{array}{c}\text { Año de } \\
\text { entrega }\end{array}$ & Distinción & Entrevistadas & $\begin{array}{l}\text { Lugar de trabajo al momento } \\
\text { de recibir el premio }\end{array}$ & Disciplina \\
\hline 2016 & Premio & $\begin{array}{l}\text { Fabiana } \\
\text { Gennari }\end{array}$ & $\begin{array}{l}\text { Gerencia de Investigación } \\
\text { Aplicada/ Comisión } \\
\text { Nacional de Energía Atómica }\end{array}$ & Química \\
\cline { 2 - 6 } & Beca & $\begin{array}{l}\text { María } \\
\text { Fernanda } \\
\text { Villanueva }\end{array}$ & $\begin{array}{l}\text { Instituto de Química y } \\
\text { Metabolismo del Fármaco/ } \\
\text { ConICET-Universidad } \\
\text { Nacional de Buenos Aires }\end{array}$ & Farmacia \\
\hline \multirow{2}{*}{ Premio } & Paula Casati & $\begin{array}{l}\text { Centro de Estudios } \\
\text { Fotosintéticos y Bioquímicos/ } \\
\text { ConICET-Universidad } \\
\text { Nacional de Rosario }\end{array}$ & Biotecnología \\
\cline { 2 - 6 } & Beca & $\begin{array}{l}\text { María } \\
\text { Alejandra } \\
\text { Molina }\end{array}$ & $\begin{array}{l}\text { Departamento de Química } \\
\text { de la Facultad de Ciencias } \\
\text { Exactas, Fisicoquímicas y } \\
\text { Naturales de la Universidad } \\
\text { Nacional de Río } \\
\text { Cuarto-Córdoba }\end{array}$ & Biología \\
\hline
\end{tabular}

Fuente: elaboración propia.

\section{Las motivaciones de las participantes premiadas}

De acuerdo con la literatura sobre premios, explorar las motivaciones de los participantes constituye un punto relevante al momento de evaluar el diseño —o rediseño— del mismo, así como de las políticas destinadas a mejorar la situación de las mujeres (Bays et al., 2009). Por lo general, las motivaciones se vinculan a los beneficios no monetarios y monetarios de la participación. En el caso del Premio L'Oréal-UNESCO-CONICET, la mayoría de las entrevistadas reconoció que el principal motivo para presentarse fue la posibilidad que representa el premio de obtener financiamiento. Independientemente de la disciplina de procedencia, el monto de dinero que acompaña la entrega de una placa de distinción constituye la razón central para participar. Nociones como "subsidio", "apoyo importante", "incentivo bueno para hacer investigación" y "dinero" fueron centrales al momento de explicar el motivo de la presentación. Adicionalmente, las premiadas mencionaron el valor del reconocimiento que implica 
ser distinguida, el prestigio que representa el premio y la situación de tener un proyecto en marcha para desarrollar. Asimismo, la mayoría de las entrevistadas destacó que existen diferencias entre recibir un subsidio de investigación y este tipo de premios al señalar que, además del reconocimiento, el galardón tiene un prestigio asociado y una difusión muy distinta a la de un subsidio. De manera conjunta, la rendición y las condiciones para hacer uso del dinero son bastante más flexibles que las de un subsidio de investigación tradicional.

Por su parte, una de las becarias destacó, puntualmente, que la beca representaba el único subsidio al que podía presentarse. Por ello agregó que:

[...] el dinero que me dieron equivalía a un PICT joven ${ }^{19}$, entonces me permitió desarrollar todo el proyecto de investigación... me dio una muy buena oportunidad de independencia económica.

De lo anterior se desprenden varias cuestiones. Por un lado, se pone de manifiesto la importancia de que el premio implique, no solo reconocimiento y prestigio, sino una suma de dinero para llevar adelante una investigación. En este sentido, desde la literatura, se afirma que existen sesgos de género en los subsidios a la investigación (Comisión Europea, 2009), es decir que los hombres tienen mayores tasas de adjudicación que las mujeres en las competencias por subsidios a proyectos. Al indagar en las motivaciones de quienes han sido distinguidas, se advierte que, sin ser un objetivo declarado, el Premio L'Oréal-UNESCO-CONICET puede entenderse como una posibilidad de sortear dicho desequilibrio. En este contexto, el hecho de que el financiamiento sea la principal motivación podría indicar una brecha de género respecto a las políticas de subsidios a la investigación en el país. Por otro lado, el premio es visto como diferente a un subsidio - tradicional_ ya que está destinado solo a mujeres y posee condiciones de rendición flexibles que ocupan menor tiempo y permiten emplear mejor el dinero.

$19 \quad$ Ver nota al pie 17. 
En un diagnóstico realizado hace ya algunos años en Argentina (Baringoltz \& Posadas, 2007), se encontró que en un universo compuesto por cinco convocatorias entre los años 2000 y 2005, hay diferencia a favor de los investigadores varones en el otorgamiento de uno de los principales instrumentos de financiamiento en el país: los PICT a investigadores formados aunque, de una convocatoria a otra, se observó una reducción de la diferencia porcentual entre géneros hasta llegar a valores de paridad en la convocatoria de 2005. Sin embargo, el cálculo de indicadores desagregados por disciplina mostró notables diferencias en cuanto a la situación de género. Así, se observaron disciplinas masculinizadas, feminizadas o en paridad de género. Por ejemplo, en los PICT para investigadores formados, de trece disciplinas involucradas, tres se encontraron en paridad de género, diez estaban masculinizadas y no existía ninguna feminizada. Por su parte, en los PICT para jóvenes investigadores, de las trece disciplinas consideradas, cinco se encontraban en paridad, cinco estaban masculinizadas y tres feminizadas. Habrá que profundizar en el diagnóstico para conocer la situación una década después.

\section{Los efectos en las premiadas: estar en la mira, en el foco de la atención}

Si bien existen escasas evaluaciones o trabajos académicos que exploren los impactos de la implementación de los premios, ya que se torna complejo discernir si los efectos son producto de haber recibido una distinción o de otro factor, a partir de las respuestas de las entrevistadas respecto a los efectos que ellas percibieron, luego de ser premiadas, puede afirmarse que la principal consecuencia ha sido el aumento de la visibilidad del trabajo de cada ganadora, del equipo y de la institución a la que pertenecen en su conjunto. La mayoría de las entrevistadas mencionó haber sido contactada por medios de comunicación masiva, haber dado entrevistas a radios, canales de televisión y prensa gráfica de importante llegada popular, producto de haber sido premiadas. En palabras de dos de las ganadoras:

—El impacto más grande, me parece a mí, fue desde el punto de vista de la visibilidad... el Premio L'Oréal te abre la posibilidad 
de mostrarle a la sociedad lo que haces, llegás a un público [al] que normalmente no podés llegar, o no es fácil.

-En mi caso, terminaron entrevistándome desde revistas que están en todos los lugares, como el odontólogo o la peluquería. Entonces a mí me empezaron a conocer mucho más, no solo desde el mundo científico sino de la gente.

Entre las entrevistadas surgió, además, que la visibilidad que adquirieron como investigadoras y la temática de su proyecto no solo se produjo en el ámbito científico, sino también en el sector productivo y en la sociedad en general. En sus respuestas subrayaron que:

— Se visibiliza la ciencia en sectores de la sociedad que no están habituados.

—Tuvimos más visibilidad, uno queda más en la mirada. Me hicieron bastantes notas a nivel local, en radios, en canal, no solo a mí sino a varias mujeres del instituto.

-Dos empresas se comunicaron con nosotros y eso fue súper importante porque a veces a nosotros, desde la universidad, conectarnos con las empresas nos es difícil. El premio sirve de puente y [para] que la gente conozca lo que estamos haciendo. Esta gente de las empresas escuchó en entrevistas y leyó las notas del diario y ahí hicimos un trabajo con ellos. Yo creo que la difusión es muy importante.

Además de la visibilidad, dos de las premiadas mencionaron el impacto que tuvo el premio en cuanto a "generar una responsabilidad porque ves que entonces hay muchas mujeres que empiezan a sentir que ellas también pueden seguir una carrera científica..." y a "tomar conciencia del tema de la mujer en la ciencia. No era algo que yo supiera. Empecé a leer muchísimo a partir de haber ganado el premio".

El tema de la visibilidad ha sido señalado como un problema en la literatura sobre ciencia y género desde los comienzos de la indagación sobre el tema. Incluso, en la década de los ochenta, las historias de mujeres científicas eran casi inexistentes. Las explicaciones de este "olvido" son varias (Rossiter, 1993). Por un lado, pudo constatarse que 
las mujeres se distribuían normalmente en las posiciones que requerían menos entrenamiento y menor poder (asistentes de laboratorios por ejemplo), lo que conducía a que sus nombres fueran raramente recogidos en las memorias. Esto hizo que no fueran un objetivo que atrajera la atención de los historiadores de la ciencia; por otro lado, estaban inmersas en un paradigma en el que la ciencia era descrita como la empresa de unos pocos "genios" aislados (Sanz González, 1998). Respecto al tema de los premios y la cuestión de género, un caso muy conocido es el de Rosalin Franklin, que se convirtió casi en un ícono del feminismo debido a que no le fue reconocida su contribución al descubrimiento de la estructura del ADN, por lo que Watson y Crick recibieron el premio $\mathrm{Nobel}^{20}$.

Otro aspecto a mencionar respecto a la visibilidad como efecto del premio remite a que dos entrevistadas señalaron que ni su trayectoria como científica ni su proyecto recibieron atención y lo adjudicaron al hecho de haber ganado una mención y no el premio. Este dato pone el acento sobre los efectos de aquellos galardones que tienen jerarquía de premios (Szymanski \& Valletti, 2005). Las razones para dar un segundo puesto o mención parecen responder a incentivar de alguna manera los proyectos y trayectorias que, igualmente, destacan en el conjunto de presentaciones. No obstante, el malestar o rechazo provocado en las ganadoras de menciones pueden generar un efecto negativo y causar desmotivación, subvaloración y resistencia a participar de futuras competencias.

\section{La opinión de las ganadoras sobre la participación de la mujer en la ciencia}

A partir de la constatación de que el aumento de la visibilidad ha sido un aspecto mencionado por la mayoría de las entrevistadas como un efecto importante luego de haber recibido el premio, la mención o la beca, se considera pertinente conocer la visión sobre el tema que

\footnotetext{
20 Para mayores detalles del episodio ver, Rodrigues da Silva, M. (2010). As controvérsias a respeito da participação de Rosalind Franklin na construção do modelo da dupla hélice. Scientiae Studia, 8(1), 69-92.
} 
poseen las ganadoras, ya que su exposición mediática las convierte en un referente o modelo y, por lo tanto, sus reflexiones también adquieren relevancia. Retomando el principal objetivo de este instrumento de política de mejorar la situación de la mujer en la ciencia, en un segundo bloque de preguntas se exploró la visión de las ganadoras respecto a la problemática de acuerdo a sus experiencias como científicas. Allí, algunas opiniones fueron dispares aunque, en general, hubo coincidencias de énfasis en ciertas cuestiones.

Si bien la mayoría de las entrevistadas señaló una mejora en la situación de la mujer en la ciencia en Argentina, en los últimos diez años, todas marcaron que existe, al menos, un desequilibrio en la participación de la mujer en cargos decisorios en organismos científicos y en las categorías superiores de la carrera en investigación. Tres de las entrevistadas mostraron un conocimiento informado sobre la temática al mencionar nociones específicas de la literatura sobre género y ciencia (Yáñez, 2016) como: leaky pipeline, glass ceiling y discriminación positiva. Si bien solo cinco de las diecinueve entrevistadas expresaron haber sufrido obstáculos por ser mujer durante el desarrollo de su carrera, las restantes mencionaron situaciones de colegas y reconocieron que quizás haya habido escenarios de discriminación hacia ellas, pero no tuvieron conocimiento de esto.

Respecto a las causas de la disparidad de la participación de las mujeres en la ciencia, las primeras razones mencionadas por todas las entrevistadas se centraron en la cultura, "el subconsciente", "la sociedad machista", "la crianza", "la religión". Un segundo factor mencionado — asociado al anterior — fue la mayor dedicación de la mujer a la familia y a las cuestiones del hogar como una demanda cultural o de la sociedad, causas mencionadas en la literatura (Misra et al., 2012; Vohlídalová, 2017): "la vida familiar es de altísima demanda", "porque la mujer todavía sigue cargando con mucho peso de la familia, más que el hombre, entonces se hace cargo de actividades que deberían ser más compartidas", "la mujer ha tenido que cargar con muchas tareas del cuidado familiar". De acuerdo a dos entrevistadas, la científica actual se ha convertido en una mujer "multitasking", en "la súper mujer". 
En relación con otro orden de factores, se consultó a las premiadas respecto al papel de los organismos científicos como actores activos en la promoción de una mayor participación de las mujeres en ciencia. La totalidad de las entrevistadas consideró la importancia de que los propios organismos de promoción de la ciencia implementen políticas específicas para lograr la equidad de género. Entre las acciones concretas o temas que se repitieron se encuentran la introducción de la licencia por paternidad, la creación o ampliación de cupos en guarderías y jardines asociados a institutos, laboratorios o universidades, la posibilidad de subsidios de investigación solo para científicas, la constitución de oficinas especiales para resolver cuestiones de discriminación por género, la incorporación de la cuestión de género entre los criterios de evaluación. Una iniciativa que la mayoría de las entrevistadas manifestó rechazar, de manera contundente, fue la introducción de cuotas o cupos femeninos en los organismos científicos debido a que consideran que restaría valor al mérito o excelencia científica.

Por último, con relación al papel de la comunidad científica en la búsqueda de la incorporación de una perspectiva de género en sus actividades, las respuestas fueron muy diversas. Una porción de entrevistadas señaló la posibilidad de incidir en la organización de congresos y en las evaluaciones para becas o subsidios al ocuparse de que la proporción de mujeres y hombres sea aproximadamente paritaria. Algunas de las entrevistadas integran asociaciones u organismos científicos a nivel internacional y es allí donde la paridad de género se convierte en un imperativo más fuerte que a nivel nacional; señalaron que "si no hay mujeres no estás dentro de estándares internacionales", "en muchos congresos y simposios internacionales te piden que haya mujeres... son muy cuestionados los workshops en donde han puesto todos hombres". Otra de las entrevistadas contó que:

[...] nos tocó organizar un curso internacional y pedimos subvención a organismos internacionales y, nosotros inconscientemente organizamos las disertaciones y eran todos varones... a pesar de que había mujeres con los mismos antecedentes para ser también disertantes. Desde el organismo internacional nos pidieron que 
incorporáramos mujeres en nuestro programa, entonces lo hicimos y a partir de allí tenemos en cuenta la cuestión de género en los eventos que organizamos. A igualdad de calidad académica escogemos mujeres y hombres.

Otro conjunto de entrevistadas apuntó a la difusión de un modelo de científica entre las más jóvenes, entre las niñas o adolescentes y al trabajo en las escuelas. Varias de las entrevistadas resaltaron la importancia del trabajo con los jóvenes y de la motivación en la instancia escolar para estudiar carreras en ciencias e ingenierías. En sus palabras: "hay que apuntar a las chicas en edad escolar porque creo que la raíz de todo esto empieza en la infancia donde las mujeres no son estimuladas para seguir ciencia". En la literatura sobre género existe un conjunto de trabajos (Yáñez, 2016) que ponen énfasis en la implementación de este tipo de medidas, con el objetivo de aumentar la participación de la mujer en la etapa de la elección de una carrera universitaria.

\section{Conclusiones}

Este artículo buscó brindar un análisis del Premio L'Oréal-UNESCO en Argentina a partir de los testimonios de sus ganadoras. Se propuso revelar su dinámica y contribuir a una mejor comprensión de los efectos de los premios en la promoción de la participación de las mujeres en ciencia. También intentó resaltar las ventajas y debilidades de los premios en determinadas circunstancias y proporcionar información para el diseño e implementación de premios efectivos. Asimismo, del estudio surgen algunas consideraciones metodológicas para profundizar en futuras investigaciones empíricas sobre los premios destinados, exclusivamente, a mujeres.

En primer lugar, se mostró que los premios son un instrumento complejo y su estudio requiere analizar la voz de las propias ganadoras, por lo general no incorporada por la literatura que realiza análisis cuantitativos. Se considera que los premios complementan y no reemplazan a otros instrumentos de incentivos en la promoción de la CTI y que son solo una de las formas de intervención para estimular 
la participación de la mujer en la ciencia, pero son particularmente apropiados, por ejemplo, para dar visibilidad a la labor de las mujeres en ciencia; dar impulso a las jóvenes para desarrollar una línea de investigación; difundir modelos de científicas exitosas en disciplinas con pocas mujeres, y propiciar la incorporación de mujeres en instancias superiores de la carrera académica o en cargos de decisión. Los premios pueden dirigirse selectivamente a determinados tramos de la carrera científica y a disciplinas y, también, pueden aprovechar los fondos de forma significativa debido a su mayor flexibilidad en la aplicación como en la rendición. Implican, sin embargo, algunos efectos no esperados respecto a otros instrumentos más tradicionales y a su uso rutinario y/o desafían definiciones y debilitan el poder de incentivo del mecanismo como en el caso de las menciones. De allí que la implementación exitosa de los premios requiera de un diseño adecuado a las motivaciones de las participantes y al contexto nacional.

Un dato a destacar del premio estudiado es que, si bien el efecto central identificado por las propias ganadoras es el aumento de la visibilidad que obtuvieron, el premio carece de un trabajo de difusión del papel de la mujer en la ciencia. En general, el mensaje que se ha transmitido en los medios de comunicación de los propios patrocinadores del premio y en los medios masivos se ha centrado en el tema de investigación de las premiadas, antes que en su carrera científica y en el rol como mujeres en el desarrollo de la ciencia.

Asimismo, se consideró relevante explorar la visión de las ganadoras sobre la participación de la mujer en la ciencia en Argentina, en particular, en la disciplina de trabajo y con base en la experiencia personal. De sus opiniones se desprenden algunas reflexiones. Por un lado, a pesar de haber recibido un premio enmarcado en un programa que busca combatir la discriminación por cuestiones de género en la ciencia, la mayoría de las ganadoras manifestó no haber sufrido obstáculos, barreras o algún episodio de discriminación a lo largo de su carrera aunque advirtieron que, si lo hubo, no fueron conscientes o no tomaron conocimiento y afirmaron que reconocen que sí hay inequidad. Este tipo de testimonios arroja luz respecto a los objetivos fundamentales del premio: funcionar como un reconocimiento y un 
subsidio de investigación para científicas que poseen una trayectoria relevante y un proyecto de investigación de impacto para el país. Este tipo de premios no representa un reconocimiento a la labor de mujeres científicas que hayan sorteado dificultades en sus carreras, ni un premio al activismo en pos de reducir las disparidades en cuanto a la participación de las mujeres en ciencias. De esta manera, otros tipos de premios podrían complementar al existente y contribuir a impulsar grupos diferentes de científicas que hacen ciencia.

Por su parte, de acuerdo con la opinión de las entrevistadas, la principal razón del desequilibro de la participación de la mujer en la ciencia es cultural y social. Una minoría de las entrevistadas mencionó, en primer lugar, la responsabilidad de las instituciones en mejorar la situación de la mujer en la ciencia. Solo algunas subrayaron la necesidad de un mayor involucramiento de los propios organismos científicos gubernamentales. En este sentido, se observa que, incluso quienes han sido distinguidas por un premio que busca reducir la brecha de género en la ciencia, mantienen los supuestos más tradicionales sobre la problemática que se enfocan en la necesidad de que sea la propia mujer la que modifique su papel frente a su familia y a las demandas de la sociedad. La incorporación de políticas de igualdad de oportunidades en los organismos científicos requiere de la consulta con expertos y expertas pero, sobre todo, de la consulta de las partes afectadas y de las propias científicas involucradas de alguna manera en la problemática. La visión de las ganadoras de los premios L'Oréal-UNESCO-CONICET constituye un aporte relevante en este sentido y podría constituir importantes referentes de propuestas, aunque debería también contemplarse la participación de científicas de otras disciplinas.

Finalmente, a través de este artículo, se buscó contribuir a una mejor comprensión de los instrumentos de política en CTI en Argentina, específicamente, de los premios para mujeres científicas. Se introdujo un enfoque de análisis, para investigar los premios, que se centra en los tipos de incentivos y de resultados que pueden explicar el efecto de los premios como iniciativas de género. La evidencia cualitativa permitió, en este sentido, distinguir las diferencias percibidas por las entrevistadas 
entre este premio y los subsidios de investigación: el premio presenta mayor flexibilidad en las condiciones de uso de los recursos económicos y posee un tipo de difusión que genera una visibilidad masiva por fuera del ambiente científico, tanto en la sociedad en general como en sectores productivos. Además, la particularidad de estar destinado solo a mujeres lo convierte en un instrumento único en el país. Esta iniciativa merece atención especial dentro del conjunto de políticas que buscan promover la participación de las mujeres en la ciencia y requiere ser todavía más explorada y analizada desde otros puntos de vista, más allá de las ganadoras como, por ejemplo, mediante la consideración de las autoridades del propio CONICET y del Ministerio de Ciencia, Tecnología e Innovación Productiva.

\section{Referencias}

Baader, M. S., Böhringer, D., Korff, S. \& Roman, N. (2017). Equal opportunities in the postdoctoral phase in Germany? European Educational Research Journal, 16(2-3), 277-297. Doi: https://doi. org/10.1177/1474904117694624

Balafoutas, L. \& Sutter, M. (2012). Affirmative action policies promote women and do not harm efficiency in the laboratory. Science, 335(6068), 579-582. Doi: http://dx.doi.org/10.1126/science.1211180

Baptista, B. (2017). Los instrumentos de política de ciencia, tecnología e innovación en América Latina. En Ricyt (Ed.), El estado de la ciencia. Principales indicadores de Ciencia y Tecnología iberoamericanos/interamericanos 2016 (pp. 45-53). Buenos Aires: RicyT-OeI-Unesco. Recuperado el 5 de noviembre de 2017 de http://www.ricyt.org/publicaciones

Baringoltz, E. \& Posadas, P. (2007). Ciencia y tecnología en la Argentina. Diagnóstico de la situación de género (julio de 2006 - diciembre de 2007). Recuperado el 5 de noviembre de 2017 de http://www.mincyt.gob.ar/estudios/ ciencia-y-tecnologia-en-la-argentina-diagnostico-de-la-situacion-degenero-8031

Bays, J., Chakravorti, B., Goland, T., Harris, B., Jansen, P., McGaw, D. \& Tallento, L. (2009). And the winner is...: Capturing the promise of philantropic prizes. McKinsey \& Company. Recuperado el 5 de noviembre de 2017 de https:/ /www.mckinsey.com/industries/social-sector/ 
our-insights/ and-the-winner-is-philanthropists-and-governmentsmake-prizes-count

Blickenstaff, J. C. (2005). Women and science careers: leaky pipeline or gender filter? Gender and Education, 17(4), 369-386. Doi: https://doi. org/10.1080/09540250500145072

Bukstein, D. \& Gandelman, N. (2017). Glass ceiling in research: Evidence from a national program in Uruguay. Inter-American Development Bank. Recuperado el 5 de noviembre de 2017 de https://publications.iadb.org/bitstream/handle/11319/8244/ Glass-Ceiling-inResearch-Evidence-from-a-National-Program-in-Uruguay.PDF? sequence $=1 \&$ is Allowed $=y$

Carnes, M., Geller, S., Fine, E., Sheridan, J. \& Handelsman, J. (2005). NIH Director's Pioneer Awards: Could the selection process be biased against women? Journal of Women's Health, 14(8), 684-691. Doi: http:/ / doi.org/10.1089/jwh.2005.14.684

Comision Europea. (2009). The gender challenge in research funding: Assessing the European national scenes. Luxembourg: Luxembourg Press - European Commission.

Conicet. (2017). Bases del Premio Nacional L’Oréal-Unesco "Por las Mujeres en la Ciencia", en colaboración con CONICET para el mejor proyecto de investigación en "Ciencias de la Vida”. Edición 2017. Recuperado el 5 de noviembre de 2017 de http://www.conicet.gov. ar/wp-content/uploads / ANEXO-I-BASES-DEL-PROGRAMALOREAL-Premios-y-Beca-2017.pdf

Estébanez, M. E. (2012). Género y profesión en el análisis de la ciencia argentina. Versión adaptada del paper presentado en el Foro Nacional Interdisciplinario Mujeres en Ciencia, Tecnología y Sociedad FONIM. Bariloche. Recuperado el 2 de febrero de 2018 de http://www.politicascti.net/

Estébanez, M. E. (2007). Género e investigación científica en universidades latinoamericanas. Educación Superior y Sociedad, 1(1), 2-26. Recuperado el 2 de febrero de 2018 de http: / / ess.iesalc.unesco.org.ve/ess3/ index. $\mathrm{php} / \mathrm{ess} /$ article/view/24

Franchi, A., Atrio, J., Maffia, D. \& Kochen, D. (2008). Inserción de las mujeres en el sector científico-tecnológico en la Argentina (1984-2006). Arbor, 184(733), 827-834. Recuperado el 9 de diciembre de 2017 de http://arbor.revistas.csic.es/index.php/arbor/article/view/227/228 
Frey, B. S. \& Gallus, J. (2017). Towards an economics of awards. Journal of Economic Surveys, 31(1), 190-200. Doi: http://doi.org/10.1111/ joes. 12127

García, C. C. \& Morán, M. P. (2008). Permisos de maternidad, paternidad y parentales en Europa: algunos elementos para el análisis de la situación actual. En XV Encuentro de Economía Pública: políticas públicas y migración. Papeles de trabajo número 26/07. Recuperado el 26 de enero de http://www.ief.es/documentos/recursos/publicaciones/papeles_trabajo/2007_26.pdf

Giménez Elorriaga, C. \& Monike, N. (2016). Impacto de los premios en género, ciencia y tecnología: del efecto Matilda a la teoría del embudo en la creación del Premio Ada Byron a la Mujer Tecnóloga de la Universidad de Deusto. XI Congreso Ciencia, Tecnología y Género. Recuperado el 2 de febrero de 2018 de https:/ / congresoctg.ucr.ac.cr/ memoria/descargar.php?id $=21$

Gök, A. (2013). The impact of innovation inducement prizes. NESTA working paper series, No 13/18. Recuperado 26 de enero de 2018 de: www.nesta.org. $\mathrm{uk} / \mathrm{sites} /$ default/files/impact_of_innovation_inducement_prizes.pdf

Lincoln, A. E., Pincus, S., Koster, J. B. \& Leboy, P. S. (2012). The Matilda effect in science: Awards and prizes in the US, 1990's and 2000's. Social Studies of Science, 42(2), 307-320. Doi: http://doi.org/ $10.1177 / 0306312711435830$

López Sancho, M. P. (2016). Las mujeres y la ciencia: programa L’OréalUneSCO "Por las Mujeres en la Ciencia" en España. Revista 100cias@ uned, Nueva Época, (9), 176-183.

Mairesse, J. \& Pezzoni, M. (2015). Does gender affect scientific productivity? Revue Économique, 66(1), 65-113. Doi: https://doi.org/10.3917/reco.661.0065

Mason, M. A., Wolfinger, N. H. \& Goulden, M. (2013). Do babies matter? Gender and family in the Ivory Tower. London: Rutgers University Press.

Misra, J., Lundquist, J. H. \& Templer, A. (2012). Gender, work time, and care responsibilities among faculty. Sociological Forum, 27(2), 300-323. Doi: https://doi.org/10.1111/j.1573-7861.2012.01319.x

Nielsen, M. W. (2016). Gender inequality and research performance: moving beyond individual-meritocratic explanations of academic advancement. Studies in Higher Education, 41(11), 2044-2060. Doi: https:// doi.org/10.1080/03075079.2015.1007945 
Rodrigues da Silva, M. (2010). As controvérsias a respeito da participação de Rosalind Franklin na construção do modelo da dupla hélice. Scientiae Studia, 8(1), 69-92. Doi: http://dx.doi.org/10.1590/S167831662010000100004

Rossiter, M. W. (1993). The Matthew Matilda effect in science. Social studies of Science, 23(2), 325-341. Doi: https://doi.org/10.1177/ 030631293023002004

Rudman, L. A. (1998). Self-promotion as a risk factor for women: The costs and benefits of counterstereotypical impression management. Journal of Personality and Social Psychology, 74(3), 629. Doi: https://doi. org/10.1037//0022-3514.74.3.629

Salamon, L. (Ed.). (2002). The tools of government: A guide to the new governance. Oxford: University Press.

Sanz González, V. (2005). Una introducción a los estudios sobre ciencia y género. Argumentos de Razón Técnica, 2005(8), 43-66.

Sarthou, N. F. (2015). Los instrumentos de política como enfoque de análisis de los sistemas de pago al mérito: contribuciones analíticas a partir del caso argentino. Perfiles Educativos, 37(149), 150-168.

Sarthou, N. F. (2018). Los instrumentos de la política en Ciencia, Tecnología e Innovación en la Argentina reciente. Revista Trilogía Ciencia Tecnología Sociedad, 10(18), 97-116. Doi: http://doi.org/10.22430/21457778.666

Silver, J., Bhatnagar, S., Blauwet, C., Zafonte, R., Mazwi, N., Slocum, C. \& Tenforde, A. (2017). Female physicians are underrepresented in recognition awards from the American Academy of Physical Medicine and Rehabilitation. PM\&R, 9(10), 976-984. Doi: http://doi.org/10.1097/ PHM.0000000000000792

Suárez, D. \& Fiorentin, F. (2018) Federalización y efecto. Mateo en la politica científica. El caso del PICT en la Argentina (2012-2015). Documento de trabajo CIECTI No. 12. Recuperado el 5 de Julio de 2018 de http:/ /www.ciecti. org.ar/wp-content/uploads/2018/06/DT12-Federalizacion_Mateo_PICT.pdf

Szymanski, S. \& Valletti, T. M. (2005). Incentive effects of second prizes. European Journal of Political Economy, 21(2), 467-481. Doi: hhtp://doi. org/10.1016/j.ejpoleco.2004.07.002

UNESCO. (2007). Estrategia a plazo medio para 2008-2013.

UNESCO. (2014a). Estrategia a plazo medio 2014-2021. 
UNESCO. (2014b) Priority gender equality action plan - 2014-2021. Recuperado de http://unesdoc.unesco.org/images/0022/002272/227222e.pdf

UNESCO. (2015). UNESCO science report: Towards 2030. Recuperado el 15 de febrero de 2018 de https://es.unesco.org/node/275037

Van Arensbergen, P., van der Weijden, I., y van den Besselaar, P. (2012). Gender differences in scientific productivity: A persisting phenomenon? Scientometrics, 93(3), 857-868. Doi: http://doi.org/10.1007/ s11192-012-0712-y

Vedung, E. (2011). Policy instruments: Typologies and theories. En M. Bemelmans-Videc, R. Rist \& E. Vedung (Eds.), Carrots, sticks, and sermons: Policy instruments and their evaluation (pp. 21-58). New Brunswick: Transaction Books.

Vohlídalová, M. (2017). Academic couples, parenthood and women's research careers. European Educational Research Journal, 16(2-3), 166-182. Doi: https://doi.org/10.1177/1474904116668883

Weir, P., Leach, J., Gamble, J. \& Creedy, D. (2014). Twenty-First Century Minerva: Are there career impacts for women who receive a "Women in Science" fellowship. International Journal of Gender, Science and Technology, 6(2), 197-208.

Yáñez, S. (2016). Trayectorias laborales de mujeres en ciencia y tecnología. Barrerasy desafios. Un estudio exploratorio. Documento de trabajo No. 2. Recuperado el 5 de noviembre de 2017 de http:/ /www.flacsochile.org/publicaciones/ trayectorias-laborales-de-mujeres-en-ciencia-y-tecnologia/ 